\title{
Los aspectos socioculturales del área de lengua extranjera y el programa linguapax
}

\section{Carmen Guillén, Ana Isabel Alario y Paloma Castro}

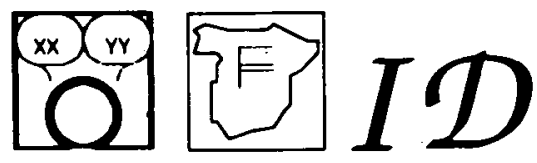

La enseñanza de lenguas extranjeras está experimentando un «enriquecimiento curricular» muy positivo. De considerarse como un simple instrumento vebicular de conocimientos o comunicación, las lenguas extranjeras están pasando a conectarse con el gran problema social y cultural de conocer otros pueblos, de conocer el mundo. El objetivo-medio de la globalización (con ciencias sociales principalmente pero no sólo) se convierte asi en uno de los puntos fuertes de la enseñanza de otras lenguas.

\section{EL APRENDIZAJE DE LENGUAS EXTRANJERAS HOY}

Las directrices de la UNESCO en política de enseñanza que señalaban como uno de los fines principales de la educación el fomentar la comprensión de la vida económica, social y cultural de los pueblos así como la solidaridad y cooperación internacionales han influido en la política educativa de muchos países, al integrar de forma significativa la enseñanza de lenguas extranjeras en los diferentes sistemas educativos. En concreto hoy nos podemos referir a nuestra reciente Ley de Ordenación General del Sistema Educativo (1).

El conocimiento y uso de lenguas extranjeras «... ya no es hoy día un lujo...» (2), se ha convertido en una necesidad generalizada al ser el instrumento de promoción de la comprensión y solidaridad entre los pueblos antes citadas, instrumento de apertura al exterior que hará viable el desarrollo de proyectos comunes por la posibilidad de dialogar, de entrar en contacto con personas y culturas de otros países.

Esta necesidad ha sido declarada de forma insistente a través de numerosas iniciativas. $Y$, con la perspectiva de la dimensión europea e internacional de la educación, se viene preconizando incluso un plurilingüismo al considerar que el monolingüismo es actualmente un inconveniente. 


\section{El programa LINGUAPAX}

De entre las numerosas acciones e iniciativas promovidas en este sentido, queremos destacar el programa LINGUAPAX (3) desarrollado en el marco de la Comisión de las Comunidades Europeas dentro del proyecto «La Juventud con Europa» en un intento - y transcribimos textualmente- de «poner en relación la enseñanza de las lenguas extranjeras con la promoción de la comprensión y de la solidaridad entre los pueblos y de la paz».

Se trata de unir el progreso en el uso de la lengua con el descubrimiento del valor de la solidaridad en una combinación óptima. Para su consecución se piensa en recomendaciones que orienten la elaboración y concepción de materiales de enseñanza menos etnocentristas, en programas de sensibilización ecológica y de educación para la paz, con la colaboración activa de toda la escuela. Existe ya una red de escuelas asociadas con la Unesco dispuestas a trabajar en este campo (4).

Pero es evidente que, en una situación más «modesta», es decir, el profesor confrontado a su grupo-clase, también todo esto se debe intentar y puede lograrse, porque se asuma esa responsabilidad.

\section{EL MARCO EDUCATIVO ACTUAL: OBJETIVOS Y CONTENIDOS EN LAS DISPOSICIONES OFICIALES}

Al plantearse la selección y estructuración de contenidos en la renovación de la enseñanza de lenguas extranjeras, la LOGSE ha efectuado un cambio, al menos en su formulación, al abordar el lenguaje desde un punto de vista funcional.

Las Disposiciones Oficiales (5) para las distintas etapas y tramos educativos -que expresan en esta área dos finalidades básicas: la instrumental y la intelectual general, de estructuración mental y representación del mundo- apuntan a proporcionar al alumno una competencia comunicativa por el conocimiento de la lengua como medio de expresión y comunicación, bloques temáticos 1 y 2 en Educación Primaria, 1, 2 y 3 en Secundaria y Bachillerato, así como hacerle entrar en contacto con la sociedad, la mentalidad, usos y costumbres de la comunidad lingüística extranjera, bloque temático enunciado como: aspectos socioculturales (número 3 en Educación Primaria, y número 4 en Secundaria), y conocimiento sociocultural en Bachillerato (núcleo temático 4).

La opción metodológica prescrita en los D.C.B., el denominado enfoque comunicativo, se propone tratar en este último bloque citado los aspectos más cercanos a los alumnos y a su propia experiencia (6) - los cuales se describen bajo el epígrafe hechos, conceptos y principios - mediante procedimientos como el reconocimiento, el análisis y la comparación, la utilización contextualizada de reglas y hábitos de conducta, entre otros, que vienen a reclamar el uso de materiales auténticos, los denominados documentos auténticos por cuanto que, procedentes de diversas fuentes, no fueron fabricados para el aula y son considerados como referentes directos de la realidad.

Y sucede que esa realidad se propone las más de las veces de forma superficial y tópica, por un reduccionismo que promueve inconscientemente una cultura «residual», centrada en elementos más bien de carácter accidental, a modo de estereotipos que representan los hechos de forma simplista e incluso con efectos 
contraproducentes por su carácter contrastivo; cuando y además no debemos olvidar que las lenguas - unas más que otras - son «vehículos pluriculturales», ya que hoy el mundo de habla inglesa o francesa o de cualquier otra lengua no excesivamente minoritaria es algo mucho más complejo.

Pluriculturalidad que ha llevado en el plano teórico a una revisión crítica del concepto antropológico de cultura, para liberarla de una dimensión estática hacia la autonomía de la persona, la capacidad de situarse en el mundo, la capacidad para comunicarse con los demás, la capacidad de participar en la sociedad. En este sentido es relevante la definición de cultura que en su día propuso C. Camilleri utilizando términos tales como actitudes, representaciones y comportamientos (7).

Todo ello es interesante considerarlo, desde la perspectiva de las finalidades de la educación y desde la perspectiva de los objetivos generales y específicos de área (8), de forma que en la situación de enseñanza/aprendizaje se haga patente el pluralismo en que vivimos, lo cual incide y matiza toda acción pedagógica: la enseñanza de la lengua extranjera deberá acompañarse de una familiaridad con esta diversidad. Se trata de un conocimiento comprensivo de las diferencias más que de un conocimiento contrastivo, por la promoción activa de esa comprensión (9).

Obviamente, esto es difícil, pero como nos indica M. Sigúan, esta dificultad «no autoriza a renunciar al objetivo propuesto». Objetivo considerado en sus tres dimensiones: psicológica de sensibilización, cognitiva de conocimiento efectivo y práctica de comportamiento y actitudes.

\section{LA DIMENSION HUMANISTICA DE LA LENGUA, GUIA DE CONTENIDOS}

De estas consideraciones se desprende la idea de que la enseñanza/aprendizaje de las lenguas extranjeras no debe plantearse con unos objetivos estrictamente utilitarios e inmediatos.

Bien es cierto que los escolares accederán a la lengua en sus dimensiones formal e instrumental, pero además y en una doble vertiente se les debe habituar a que la utilicen para recibir y tratar información que no sea la propia lengua, e interesarles en conocer - y aquí reproducimos las palabras introductorias de M. Siguán en la reunión de Sitges, LINGUAPAX II: «... el mundo en su conjunto, en su diversidad humana y cultural y los problemas que le aquejan y que a todos nos afectan», lo cual traduce la atención a la dimensión humanística de la lengua.

El aprendizaje de la lengua debe satisfacer diversas finalidades y entre ellas las de orden social y humano que permitan el reconocimiento y la comprensión de otras culturas, en torno a tres conceptos:

- Comprensión, que se mide en función del saber del alumno y de su percepción de los valores y puntos de vista de la comunidad lingüística extranjera.

-Actitud, en función del grado de etnocentricidad respecto del otro país.

-Evolución, entendiéndose como efecto combinado del aprendizaje de la lengua y de los factores externos.

De forma que ese conocimiento no puede ni debe resolverse por lo anecdótico o lo folklórico, reducido en la práctica pedagógica a la presentación de unas 
escenas de la vida cotidiana, sino que debe incluir otros elementos que permitan desarrollar en los escolares un interés, una valoración e interpretación conducentes a la comprensión y al respeto por el patrimonio cultural y las claves socioculturales, abordando el conocimiento de esos productos culturales, de la situación económica, de los problemas psicológicos, la historia, la política, etc., como intercambio, interacción, apertura y solidaridad objetiva, reconocimiento de los válores, de los modos de vida, de las representaciones simbólicas, etc., valorando la diferencia/variedad cultural como un enriquecimiento que permita desarrollar múltiples potencialidades, por el encuentro, el contacto.

Se trata de abordar como objetos pedagógicos aquellos aspectos que $\mathrm{L}$. Porcher denomina «universales-singulares», como temas presentes en todas las sociedades y en todas las culturas, pero que cada sociedad y cada cultura vive de forma particular.

Todo aquello que entronca con las experiencias de los escolares, por vivido o por conocido y no necesariamente en la institución educativa - puesto que existe la «escuela paralela» según acepción de G. Friedmann-, ya que se encuentran, hoy más que nunca, sometidos a una abundate información desde los mass-media y en particular desde la TV con escenas internacionales de política, guerra y un largo etcétera al que se debe dar un contenido educativo mediante estrategias apropiadas.

Los contenidos de enseñanza se organizarán en torno a cuestiones como el medio ambiente urbano, social, natural y cívico, las relaciones sociales, la alimentación, los transportes, los medias: TV, canción..., el amor, la libertad, etc. Estas cuestiones, que por otra parte suelen ser tratadas por diferentes disciplinas, aparecen recogidas en el material de referencia que el mercado editorial pone a disposición del profesorado, incluso profusamente, Anexo, y sobre el cual corresponde efectuar una selección siempre adecuada a las características del grupo-clase, de los intereses, motivaciones y edades, para formar un conjunto en interrelación con los soportes y recursos.

\section{PARAMETROS METODOLOGICOS PARA UNA PRACTICA EDUCATIVA: ACCION, EXPERIENCIA Y CREATIVIDAD}

El enfoque comunicativo, en la óptica del programa LINGUAPAX, debe pues situarse en un triángulo de exigencias, sirviendo a objetivos psicológicos, cognitivos y pragmáticos, que deben ser tratados de forma coherente y global.

De esta manera y por las consideraciones anteriormente expuestas, los aspectos socioculturales quedan localizados en el vértice de los objetivos de orden psicológico, por cunato que la cultura puede situarse entre lo intelectual y lo afectivo, interesando al sistema psicoafectivo que estructura y orienta los instintos y construye una representación o visión del mundo.

En este orden de cosas, si nos atenemos a la idea de lo intercultural, en la cual predomina el criterio cualitativo sobre lo cuantitativo, ésta nos aparece definida por un contenido (aunque no únicamente) y en el ámbito pedagógico debe ser no sólo un cambio de reflexión y de teorización sino también de acción, de práctica, como una forma de proceder, de analizar fenómenos y actividades humanas puesto que se trata de una realidad social. Es por lo que la atención a la dimensión humanística de la que hablamos requiere el desarrollo de situaciones experienciales, situando a los escolares en un nivel de estrategias de inte- 
rrogación y de comprensión que les permita poner en juego su experiencia mediante discusiones, «jeux de rôle», simulaciones, juegos de percepción y comuncación, etc.

Lo cual nos conduce a estimar además que, si metodológicamente la enseñanza/aprendizaje ha de estar orientada hacia la acción, debemos recurrir al concepto de creatividad - por cuanto que significa adaptación, construcción, evolución, imaginación, interiorización, etc.-, situándonos en una perspectiva social y utilitaria (10). Creatividad como proceso de construcción instrumental (de orden instrumental y/o comportamental), destinado a resolver un problema, de forma que los ejercicios de creatividad - cuya eficacia reside en el grupocontribuyan a desarrollar, por una parte, aquellas aptitudes específicas que tienen por objeto el conocimiento del mundo, por el sentido de lo real, la atención a los otros y, por otra parte, aquellas que permiten juzgar y transformar el mundo por la capacidad de adaptación, de análisis y crítica, por la capacidad analógica, que no identidad y de abstracción, etc.

FIguRA 1

\section{OBJETIVOS \\ PSICOLOGICOS}

La lengua en una dimensión humanística: conocimiento representativo/existencial.

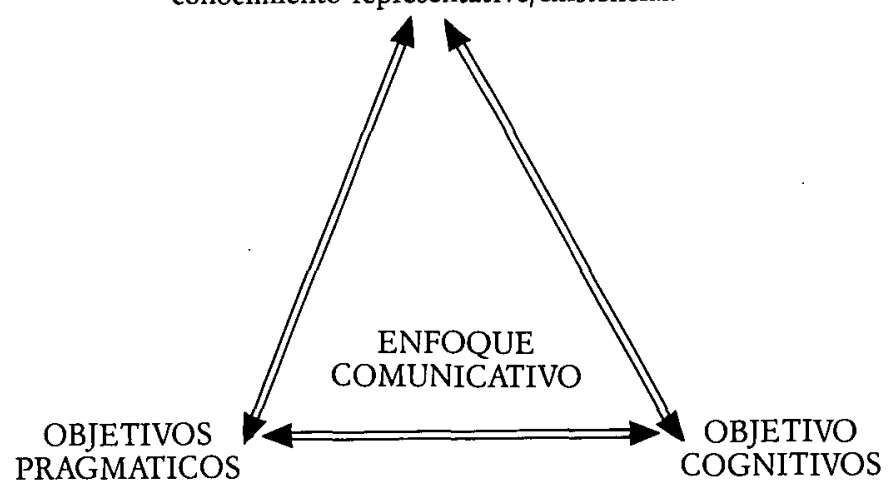

La lengua en una dimensión instrumental: conocimiento operativo

La lengua en una dimensión formal: conocimiento efectivo.

Esquema de los «vertices de atención» y sus interrelaciones desde el enfoque comunicativo.

\section{Tipología de ejercicios}

La tipología de ejercicios que exponemos aquí señala de alguna forma el itinerario de las actividades a desarrollar.

Interesa como requisito previo el desarrollo de determinados ejercicios para la constitución de los grupos, de lo que se derivará la necesaria seguridad afectiva, a saber:

- ejercicios de presentación (tradicional, con preguntas, cruzada, el retrato, el juego de los oficios, los siete deseos, etc.),

-ejercicios de relaciones interpersonales (dibujo colectivo, juegos corporales, escenificaciones, mimo, etc.). 
Dichos ejercicios permitirán el desarrollo de:

Ejercicios de sensibilización y familiarización, desde situaciones de ruptura como marco operativo para plantear un problema, buscar relaciones, propiedades, etc., de forma objetiva. Se trata de desarrollar situaciones de comunicación a las que se apliquen variaciones paramétricas sociolingüísticas sobre el status de los interlocutores, la edad, el sexo, el estado afectivo, el lugar, el tiempo, el canal de comunicación, como elementos integrantes del proceso de comunicación (11). Y siempre mediante simulaciones y «jeux de rôle», por ejemplo.

Para pasar a trabajar con ejercicios de integración en la idea de una maduración y una asimilación que se traduzcan en el hecho de conceptuar y producir por el intercambio de informaciones, por los proyectos en grupo y la puesta en común, fundamentalmente.

FIGURA 2

Itinerario de las actividades a desarrollar

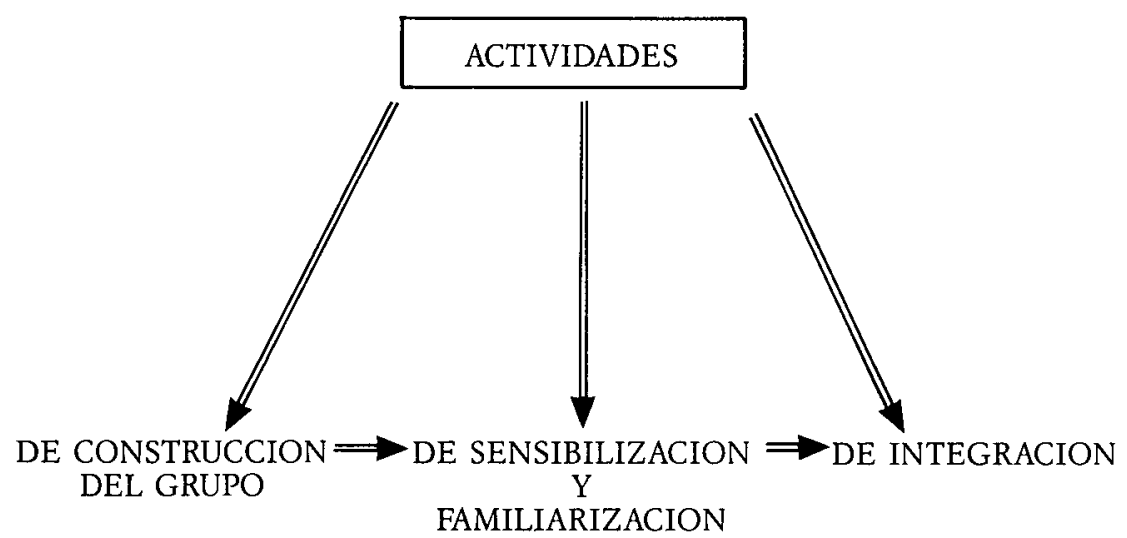

Todos ellos deben ajustarse a una serie de criterios que por su carácter consecuente expresamos a continuación, entre otros:

a) Introducirles a una información nueva y pertinente.

b) Estimularles para intercambiar opiniones sobre la información.

c) Relacionar el contenido con la existencia personal y social.

d) Adecuación al nivel lingüístico de los escolares.

El punto de partida, pues, no va a ser el manual o una disertación por parte del profesor, sino el comportamiento y la experiencia personales en un cierto número de actividades y de situaciones tanto en clase como fuera de ella, según se desprende de la definición dada por la UNESCO sobre las situaciones experienciales, en su programa educativo para el desarrollo de la comprensión internacional (12).

Actividades de enseñanza propiamente dichas, en clase, extraescolares, excursiones, intercambios, etc., unas más válidas que otras en cuanto que asignen en mayor o menor grado un papel activo al escolar en la situación de enseñanza/aprendizaje, que requieran búsqueda de ideas, análisis de una idea en un contexto nuevo, que pongan a los escolares en contacto con lo real, les den ocasión de trabajar con otros y respondan a sus motivaciones. 
En todo caso queremos a este respecto hacer referencia a las directrices de los D.C.B. en torno al diseño y tipología de tareas (13).

\section{PAUTAS PARA UNA SESION DE TRABAJO}

Sin que se trate de construir un marco prescriptivo aportamos, a modo de sugerencias, aquellas pautas que por una parte permitan generar experiencias de aprendizaje buscando una mayor adecuación a las experiencias de la vida, por la acción, la reflexión y la discusión, y que por otra parte favorezcan la integración de la evaluación del proceso de enseñanza/aprendizaje y del rendimiento escolar en sus distintos componentes; evaluación que debería comenzar por el análisis de los conocimientos y concepciones previas, dada su influencia determinante en el aprendizaje significativo.

Consecuentemente, pues, conviene alternar las fases de trabajo individual y colectivo, las fases de trabajo guiado y espontáneo, imaginativo y crítico.

Dichas fases secuenciadas en torno a:

-la percepción mediante estímulos visuales o verbales: imagen y/o texto según los niveles y que a modo de ilustraciones serán aportados para adquirir la información,

-el conocimiento,

-la interpretación/el análisis, que va más allá de la simple observación, determinando las relaciones entre los elementos,

-la comprensión,

-la discusión/apreciación, que darán lugar al enriquecimiento deseado y al reconocimiento de «otros modos» como respuestas justificadas en un contexto diferente del propio.

Fases efectuadas mediante acciones como:

- la exploración,

- la recogida de datos,

-el tratamiento de datos, dentes.

-la síntesis, reconstituyendo un todo, resultado de las operaciones prece-

Teniendo en cuenta que cada ejercicio debe definir su punto de partida y su objetivo, así como una guía o consignas de trabajo, estimamos que lo esencial reside en dos momentos que podríamos considerar claves:

1) la explicación/elucidación y

2) la comprensión dinámica, ambos conducentes a la integración de los fenómenos y actividades humanas observados en marcos de referencia más amplios y complejos. Una observación -insistimos- participante y dinámica.

La puesta en común constituirá un tercer momento que conduzca a una construcción/producción colectiva, a modo de «idea de solución» con la que se pueda concluir todo un proceso implícito de descubrimiento.

A modo de conclusión, queremos expresar nuestra convicción de que el hacer viables iniciativas como la que en este caso presenta LINGUAPAX - y que realmente no supone invertir un excesivo esfuerzo- aun en situaciones de enseñanza/aprendizaje que categorizamos como modestas -el profesor y su grupoclase - va a contribuir positivamente al bien de cada escolar, por cuanto que 
el acceso a la cultura, a lo intercultural como corolario de todas las actividades humanas está guiado por la educación.

\section{Notas}

${ }^{1}$ Ley de Ordenación General del sistema Educativo 1/1990 3 de octubre, B.O.E. 4 octubre 1990: Art. 3.3.b; Art. 14.2.e; Art. 20.2.f; Art. 27.3.4.

${ }^{2}$ Resolución de los Delegados de los Ministros: Consejo de Cooperación Cultural (1965).

${ }^{3}$ Programa «La Juventud con Europa». COM (90) 469; Decisión 88/384/CEE.

LINGUAPAX I. Enero 1986, reunión celebrada en Kiew, presidida por M. Siguán de la Universidad de Barcelona, para la declaración de principios y definición de objetivos.

LINGUAPAX II. Octubre 1987 en Sitges, en el marco del Seminario sobre «La enseñanza de las lenguas y la solidaridad internacional», organizado por el ICE de la Universidad de Barcelona.

LINGUAPAX III. Junio 1990 en Sarrebruken (R.F.A.), para el análisis de propuestas de actuaciones concretas en distintos órdenes.

${ }^{4}$ SIGUAN, M. coord. (1990). Las lenguas vivas y la educación para la paz. Barcelona, ICE Universidad de Barcelona/HORSORI. pp. 7-12.

5 D.C.B. Educación Primaria. D.C.B. Educación Secundaria. Bachillerato. Estructura y contenidos. Ministerio de Educación y Ciencia, Dirección General de Renovación Pedagógica, 1991. Reales Decretos de Enseñanzas Mínimas.

${ }_{7}^{6}$ p. 340 , D.C.B. Primaria, y pp. $454-455$, D.C.B. Secundaria.

7 CAMILLERI, C. (1989). «La notion de culture», Intercultures, 7, pp. 13-26.

${ }^{8}$ Artículo 1. g, 12, 13, 18, 19, 25.3, 26. LOGSE; B.O.E. 4 octubre 1990. Objeti$\operatorname{vos} 4$ y 7 : D.C.B.

9 Según la taxonomía de Rebecca M. VALETTE (1971), que determina entre otras las habilidades afectivas a desarrollar en el aprendizaje de otra lengua, en LEGENDRE, R. (1988). Dictionnaire actuel de l'éducation. París, Larousse. pp. 571-572.

${ }^{10}$ Por cuanto que la cultura, lo «cultural» se aborda en una perspectiva social y utilitaria aceptando la definición de MALINOWSKI, para quien la cultura es el conjunto de instrumentos de una sociedad, no sólo obras de arte, sino productos industriales o artesanales, su moral, sus instituciones, sus comportamientos, etc.

${ }^{11} \mathrm{~A}$ este respecto, remitimos al esquema ilustrativo elaborado por ABBOU, A. (1980). Approche etnométhodologique des échanges langagiers. En situation de face à face. E.L.A., 37, pp. 61-79.

${ }^{12}$ WOLSK, D. (1975). Un enseignement centré sur l'expérience. Exercices de perception, de communication et d'action. París, UNESCO.

${ }^{13}$ pp. 458-61, D.C.B. Interesa también consultar los artículos sobre actividades y tareas, publicados en el volumen monográfico: La enseñanza de lenguas extranjeras de $C L E E$, $7-8,1990$.

\section{Referencias}

Abdallah.Pretceille, M. (1986). Vers une pédagogie interculturelle. París, INRP. Publications de la Sorbonne.

Coll, C., y Orros (1992). Los contenidos en la Reforma. Madrid, Santillana.

Porcher, L. (1987). Manières de classe. París, Alliance Française/Didier.

Thevenin, A. (1980). Enseigner les différences. París, Montreal, Etudes Vivantes.

\section{Anexo}

\section{MATERIAL DE ENSEÑANZA}

Lengua Extranjera, francés:

BEACCO, J. C. et LIEUTAUD, S. (1985). Tours de France. Paris, Hachette. 
CALMY, F. et NISBETH, L (1992). Itinéraires. Paris, Hachette.

D'ORIA, D. et al (1987). Ordralphabétique, Paris, Larousse.

KIMMEL, A. (1987). Vous avez dit France? Paris, Hachette/CIEP.

LEROY, M. J. (1986). Vivre au pays. Paris, Didier.

LOUVET, R. et TOURNES, C. (1987). Seniorscope. Paris, Larousse.

MALANDAIN, L. et al (1991). Rendez-vous en France. Paris, Hachette.

MERMET, G. (1985). Francoscopie. Paris, Larousse.

QUENELLE, G. (1985). La France, j'aime. Paris, Hatier International.

.WELCOMME, G. (1986). Juniorscope. Paris, Larousse/Bayard.

\section{Lengua Extranjera, inglés:}

BROMHEAD, P. (1991). Life in Modern Britain. London, Longman.

BURROW, A.G. \& MCKECHNIE, A. (1992). Listen, Langh and Learn in London. London, Barbed Empire.

HARVEY, P. \& JONES, R. (1992). Britain explored. London, Longman.

LAIRD, E. (1986). Faces of Britain explored. London, Longman.

-(1987). Faces of the U.S.A. London, Longman.

-(1990). Welcome to great Britain and the U.S.A. London, Longman.

NOLASCO, R. \& MEDGYES, P. (1990). When in Britain. Oxford, O.U.P.

ROOM, A. (1990). An A to Z of British Life. Oxford, O.U.P.

SHEERIN, S. \& al. (1990). Spotlight on Britain. Oxford, O.U.P.

TOMSCHA, T. (1990). American Customs and Traditions. London, Longman.

\section{Los aspectos socioculturales del área de lengua extranjera y el programa linguapax Carmen Guillén, Ana Isabel Alario y Paloma Castro CL\&E, 1992, 16, pp. 83-91}

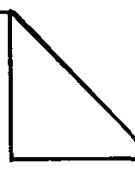

Resumen: Las autoras proponen para la enseñanza de lenguas extranjeras una acción educativa que haga viables iniciativas como la que presenta LINGUAPAX; abordando los aspectos socioculturales en una perspectiva intercultural y desarrollando en los escolares habilidades afectivas, por la promoción activa de la comprensión de las diferencias. Las consideraciones en torno al material de enseñanza, la tipología de ejercicios y la organización de las sesiones de trabajo se sitúan en el marco de las directrices de la LOGSE para las diferentes etapas y tramos educativos, los objetivos, los contenidos y el enfoque metodológico de esta área.

Datos sobre las autoras: Carmen Guillén Díaz, Ana Isabel Alario Trigueros y Paloma Castro Prieto son profesoras del Departamento de Didáctica de la Lengua y la Literatura de la Universidad de Valladolid, con docencia en la E.U. del Profesorado de E.G.B. de Valladolid. Actualmente trabajan en la investigación de las necesidades del futuro profesor de lenguas extranjeras en cuanto a la elaboración y explotación de materiales curriculares y la construcción y exploración empírica de prácticas de clase.

Dirección: Fidel Recio, 4, 5 C; 47002 Valladolid.

(C) De todos los artículos deberá solicitarse por escrito autorización de CL\&E y de los autores para el uso en forma de facsímil, fotocopia o cualquier otro medio de reproducción impresa. CL\&E se reserva el derecho de interponer las acciones legales necesarias en aquellos casos en que se contravenga la ley de derechos de autor. 